\title{
A Literature Review of Satellite Image Enhancement Methods
}

\author{
Gauri Rode $^{1}$, V. K. Shandilya ${ }^{2}$ \\ ${ }^{1}$ M. E. $2^{\text {nd }}$ year, Computer Science and Engineering, Sipna College of Engineering and Technology, Amravati, Maharashtra, India \\ ${ }^{2}$ Associate Professor in Computer Science and Engineering, Sipna College of Engineering and Technology, Amravati, Maharashtra, India
}

\begin{abstract}
Satellite images may be blurred or not clear due to many reasons. Therefore the important information in the images may be not so clear. Image enhancement can improve a satellite image which has complete information but is not visible. Image enhancement method is used to improve the quality of the image to get useful information. Many techniques have been developed to enhance the satellite images. Objective of this paper is to give information and techniques which are useful in satellite image enhancement purpose. General issues of satellite images are enhancement of gray-scale/colour image, noise, artifacts, distortion, large size, resolution, weak colour information, high frequency content and many more. Here given a discussion of the previous work of authors to deal with these issues. So that information which is visible from the image is more informative and helpful to human beings in many applications.
\end{abstract}

Keywords: Image enhancement, remote sensing, satellite image processing, image fusion, wavelet transform.

\section{Introduction}

Image processing is the processing for which the input is an image, such as a photo-graph or video frame the output we get after processing the image may be either an image or parameters related to the image. Image processing is used in many applications like Remote Sensing, Medical Application etc. There are many types of images like panchromatic (PAN), multispectral (MS), hyper spectral (HS), synthetic aperture radar (SAR) etc. Covering different parts of electromagnetic spectrum are capture by different earth observation satellites. The Satellite images have issues with their resolution, so the images which loses their high frequency contents. And they appeared as blurred image. Also many issues are related with the satellite images. Therefore the enhancement of the image is necessary to improve the visibility of the image to remove unwanted noise, artifacts, to improve contrast and to find more details. So that the some useful information is extracted to get enhance image. This is important reason behind image enhancement methods. [1,2]

The main objective of this paper is to understand the term related to the enhancement of satellite images. Image Enhancement techniques are used for making satellite images more informative and so that it readily interpretated by human eye. The meaning of enhancement is the alteration of the appearance of an image in such a way that the information contained in that image is more readily interpreted visually.

In the fig. no. 1 we have taken one example of sample image before enhancement which is taken from satellite of crop producing area, after enhancement its result visible in fig. no. 2 [11]. We can see the difference by using normal human eye. These satellite images are processed and used for crop production forecast, forest cover and type mapping, weather prediction, watershed development and monitoring, disaster management and many more. [2]

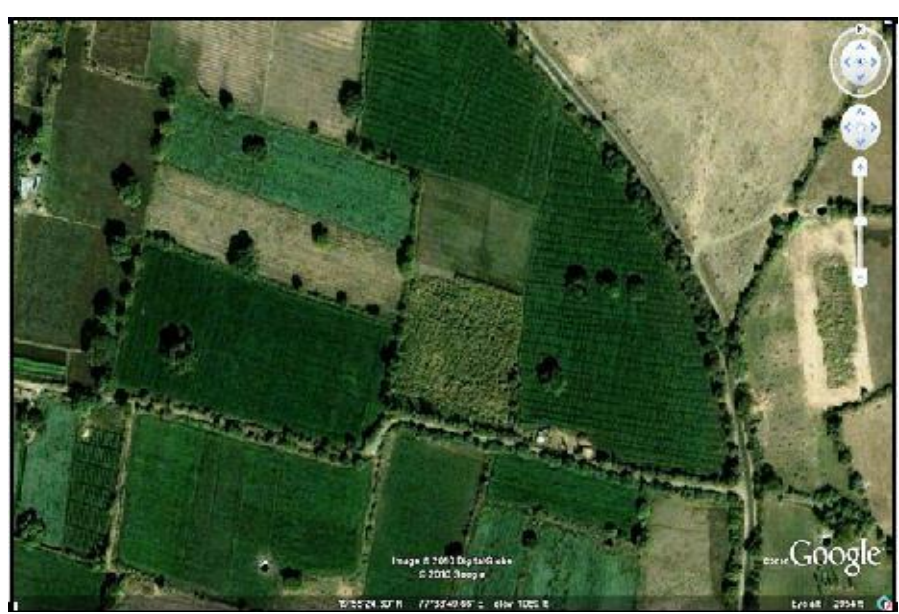

Figure 1: Sample figure before enhancement

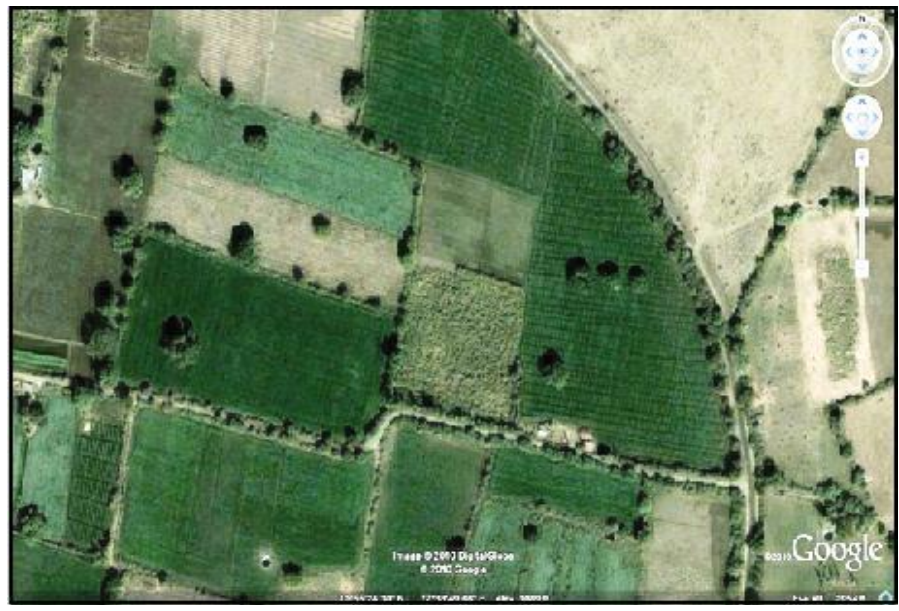

Figure 2: Sample figure after enhancement

Satellite images have been use mostly for land cover identification and classification of various features of the land surface taken from satellite. The land cover and land use mapping data which is sensed from remotely is used in different kind of applications such as environment, forestry, 


\section{International Journal of Science and Research (IJSR) \\ ISSN (Online): 2319-7064}

Index Copernicus Value (2013): 6.14 | Impact Factor (2014): 5.611

hydrology, agriculture, geology and many more. In many diverse fields the images taken from satellite are used. Therefore in different kinds of fields images have different role to perform. Image enhancement is used for making an image more interpretable for a particular kind of application, so this technique is very useful for satellite an image which is generally suitable for all kind of images. [3]

\section{Related Work}

To study the concepts of satellite image enhancement we have studied many papers. As we know the satellite images has some issues enhancement of gray-scale/colour image, noise, artifacts, distortion, large size, resolution, weak colour information, high frequency content and many more. But it is difficult to deal with all problems together. Here discussing some previous papers from which authors view and proposed methods.

P. Suganya, N. Mohanapriya et. al. [1] in this work author proposed method for satellite image enhancement which includes Haar filter for pre-processing, Multi Wavelet Transform, Interpolation Process, Inverse Process of Multi Wavelet Transform for the low resolution image. The Multi Wavelet Transform and Interpolation technique used to produce fewer artifacts. Limitation of this method is not effective method to reduce distortion and for losing of high frequency content. Also in this work describes about InterSub band Correlation Technique using Sub Bands- It used when an image is in low resolution, the image will be not clear that it is denoted as blur image. So that the frequency will also be very low and discontinuous, property is shift variant because of frequency is not continuous, the property keeps on changing. If the resolution is high, the image will be clear because of the frequency will be as much as continuous. Using Inter-Sub band correlation is we can get continuous frequency therefore the shifting property keeps invariant. Advantage of it the low resolution image will get clear and enhanced.

Arya P Unni [3] author proposed method for enhancement of satellite colour images. Using the concept of 2D Discrete wavelet transform, threshold decomposition and morphological filtering. It can differentiate unnecessary noise contents of the image and high frequency components. Also haar filter is used to filter the frequency components that are unnecessary. Also in this paper describe about Histogram Equalization technique is used to enhance images by normalizing image intensities. This method is not able to maintain average brightness level because there may be a large difference in intensity values of pixels therefore the average value is not optimum in such situations. Brightness level is either under or over saturation in the processed image. Limitation of this technique cannot be used for large images. Satellite images are large images in general so not useful in that case.

Yong Yang, Shuying Huang et. al. [5] in this work author proposed method for multi-focus image fusion and the physical meaning of wavelet coefficients, fusion technique of a discrete wavelet transform based with a new coefficients selection algorithm. Source images are decomposed by DWT method, two different window-based fusion rules are separately apply to combine the both low frequency and high frequency coefficients. Daubechies db8 as the wavelet basis for DWT based method is used. This proposed method can achieve better visual quality and objective evaluation indexes. Limitation of this method is that it does not implemented this method on multi-sensor images such as remote sensing images, medical images etc.

Pavithra C, Dr. S. Bhargavi [6] author proposed a method for fusing two dimensional multi-resolution 2-D images using wavelet transform by using the combine gradient and smoothness criterion. Basically it decompose each registered image into sub-images by using forward wavelet transform which have same resolution at that same level and different resolution at different levels. Image fusion is performed based on the high frequency sub-images and final image is obtained using inverse wavelet transform. Using the inverse wavelet transform it can reconstruct the image. This reconstructed image has information gather from all the different images sources so this is more informative. Important point is the proposed algorithm is domain independent. It can be used in different kinds of multi-modal images and also it preserves the edge information. Limitation of this it needs more space for implementation and computationally more expensive.

Wenkao Yang, Jing Wang et. al. [7] author proposed method based on principal components analysis (PCA) is a statistical technique that can convert multivariate data with correlated variables into with uncorrelated variables. The new variables are obtained as linear combinations of the existing variables. PCA technique has used for many purposes like in image encoding, image fusion, image data compression and image enhancement. In the fusion process, PCA method generates uncorrelated images. The first principal component is replaced with the panchromatic band, with one which has higher spatial resolution. Finally the inverse PCA transformation is applied to obtain the image in the RGB colour image. Limitation of it is PCA image fusion method dominant spatial information and weak colour information is often a Problem.

Anumolu Lasmika, K. Raveendra [8] author proposed a method for improving quality of satellite images. Author present a method, DWT to decompose the input image into different sub bands and apply threshold method on it. Identify the areas of the edges by using threshold decomposition method. After that the edges are sharpened by using morphological filters. This method works for sharpening and reduce the distortion of an image. Limitation of this work may be extended for other problems related to satellite images such as artifacts.

As we have seen in earlier some paragraphs the different authors have mentioned different method for satellite image enhancement. For better understanding purpose we can see the comparison and study of these all authors view and work in tabular format. Comparison of these methods is given in the table no.1. 


\section{International Journal of Science and Research (IJSR) \\ ISSN (Online): 2319-7064}

Index Copernicus Value (2013): 6.14 | Impact Factor (2014): 5.611

Table 1: Comparison of different methods

\begin{tabular}{|c|c|c|c|c|}
\hline Sr. No. & Authors & Method & Advantage & Limitation/Disadvantage \\
\hline 1. & $\begin{array}{c}\text { P.Suganya, } \\
\text { N.Mohanapriya et. al }\end{array}$ & Multi Wavelet Transform & $\begin{array}{l}\text { Reduces noise, } \\
\text { artifacts, distortion }\end{array}$ & $\begin{array}{c}\text { Used only for low resolution images, } \\
\text { Does not effective in edge } \\
\text { enhancement. }\end{array}$ \\
\hline 2. & Arya Unni & $\begin{array}{l}\text { 2D Discrete wavelet } \\
\text { transform uses haar filter }\end{array}$ & Noise removal and edge enhancement & $\begin{array}{c}\begin{array}{c}\text { Weak color information is often a } \\
\text { problem. }\end{array} \\
\end{array}$ \\
\hline 3. & $\begin{array}{l}\text { YongYang, Shuying } \\
\text { Huang et. al }\end{array}$ & $\begin{array}{c}\begin{array}{c}\text { Discrete wavelet } \\
\text { transform }\end{array} \\
\end{array}$ & Apply on multi focus image. & Does not apply on Multi sensor images \\
\hline 4. & $\begin{array}{l}\text { Pavithra C, Dr. S. } \\
\text { Bhargavi et. al }\end{array}$ & Image fusion using DWT & $\begin{array}{l}\text { Apply on multi-modal image, Used } \\
\text { for both color and grey scale images. }\end{array}$ & $\begin{array}{c}\text { Method is computationally expensive } \\
\text { and need more space. Does not apply } \\
\text { on multi resolution images. }\end{array}$ \\
\hline 5. & $\begin{array}{l}\text { Wenkao Yang, Jing } \\
\text { Wang et. al }\end{array}$ & $\begin{array}{l}\text { Principal components } \\
\text { analysis using image } \\
\text { fusion }\end{array}$ & $\begin{array}{l}\text { Effective method to sharpening of } \\
\text { image information. }\end{array}$ & $\begin{array}{l}\text { Dominant spatial information and weak } \\
\text { colour information is often a Problem }\end{array}$ \\
\hline 6. & $\begin{array}{l}\text { Anumolu Lasmika, } \mathrm{K} . \\
\text { Raveendra et. al }\end{array}$ & $\begin{array}{l}\text { DWT using } \\
\text { morphological filter }\end{array}$ & $\begin{array}{l}\text { Effective method to edge detection } \\
\text { and fine detail restoration. }\end{array}$ & Does not work on multi modal images. \\
\hline
\end{tabular}

\section{Discussion}

In this paper we have discussed about importance of satellite image enhancement also some of the techniques which are present.

Here we are discussing the problems related to the satellite images which are essential to solve. One of the important problems is sharpening of image so that it readily interpreted visually in terms of a particular need. Enhancing features which are not fully visible in the single image itself. In case of cloud cover some features may not be fully visible in one particular spectral band so there is need to fuse multiple bands. Also it is necessary to reduce noise, distortion, artifacts from the images. Image which lose high frequency content and appear as blurred image. These kind of images losses their important information, so it is essential to retrieve the information from the images. Edges contain important information in image. Therefore edge enhancement also one of the important part. We have seen in previous work that there is need to enhance the resolution of image so that we can work on multi- resolution images.

\section{Conclusion}

This paper gives the information about the enhancement of satellite images. In this paper we have also given a survey of some papers related to the satellite image enhancement and about the methods they had used in their work. Mention the comparison of the methods in the table. These methods are helpful to improve the quality of satellite images so that it becomes more informative and helpful in many applications. This paper will helpful to study the effective method for satellite image enhancement. As stated there are number of applications in which satellite images are uses.

\section{References}

[1] P. Suganya, N. Mohanapriya, B. Kalaavathi, "Satellite image resolution enhancement using multi wavelet transform and comparison of interpolation techniques", International Journal of Research in Engineering and Technology, eISSN: 2319-1163 | pISSN: 2321-7308, Volume: 03 Special Issue: 07 | May-2014.
[2] Vaibhav R. Pandit, R. J. Bhiwani, "Image Fusion in Remote Sensing Applications: A Review", International Journal of Computer Applications (0975 - 8887) Volume 120 - No.10, June 2015.

[3] Arya P Unni, "Satellite Image Enhancement Using 2D Level DWT", International Journal of Engineering Research \& Technology (IJERT), ISSN: 2278-0181, Vol. 3 Issue 3, March 2014.

[4] Dontabhaktuni Jayakumar, D. Padmashri, D. Arunakumari, "Satellite Image Fusion in Various Domains", International Journal of Advanced Engineering, Management and Science (IJAEMS), Vol1, Issue-3, ISSN: 2454-1311, June- 2015.

[5] Yong Yang1, Shuying Huang2, Junfeng Gao3, Zhongsheng Qian, "Multi-focus Image Fusion Using an Effective Discrete Wavelet Transform Based Algorithm", measurement science review, volume 14, no. 2, 2014.

[6] Pavithra C , Dr. S. Bhargavi, "Fusion of two images based on wavelet transform", International Journal of Innovative Research in Science, Engineering and Technology, Vol. 2, Issue 5, May 2013.

[7] Wenkao Yang, Jing Wang and Jing Guo, "A Novel Algorithm for Satellite Images Fusion Based on Compressed Sensing and PCA", Hindawi Publishing Corporation, Mathematical Problems in Engineering, pp. 1 - 10, Volume 2013.

[8] Lasmika, K. Raveendra, "Improving Quality of Satellite Image by Wavelet Transforming \& Morphological Filtering", International Journal of Innovative Research in Science, Engineering and Technology, ISSN: 2319 8753,Vol. 3, Issue 7, July 2014.

[9] Vinay Sahu, Dinesh Sahu, "Image Fusion using Wavelet Transform: A Review", Global Journal of Computer Science and Technology: FGraphics \& Vision, Volume 14 Issue 5 Version 1.0 Year 2014.

[10]R.Swaminathan, Dr.Manoj Wadhwa, "Satellite Image enhancement using Combination of Transform Techniques and Interpolation Methods", International Journal Of Engineering And Computer Science ISSN:2319-7242 Volume 3 Issue 4 April, 2014.

[11] Sonali P. Milmile, S. S. Godbole, "Land Classification and Crop Identification Using Gabor Wavelet Transform on Satellite Images", International Journal of Advances 
in Electronics Engineering, Vol:1 Issue:1 ISSN 2278 $215 \mathrm{X}$.

[12] K. Narasimhan, V. Elamaran, Saurav Kumar, Kundan Sharma and Pogaku Raghavendra Abhishek, "Comparison of Satellite Image Enhancement Techniques in Wavelet Domain", Research Journal of Applied Sciences, Engineering and Technology 4(24): 5492-5496, 201ISSN: 2040-7467@Maxwell Scientific Organization, 2012.

[13] Kamrul Hasan Talukder and Koichi Harada, "Haar Wavelet Based Approach for Image Compression and Quality Assessment of Compressed Image", IAENG International Journal of Applied Mathematics, 36:1, IJAM_36_1_9, Feb 07.

\section{Author Profile}

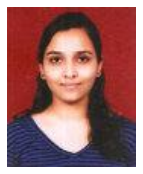

Gauri Rode received her B.E.[I.T] from H.V.P.M COET, Amravati affiliated to Sant Gadge Baba Amravati University, Amravati, Maharashtra, India in 2013. Currently pursuing her M.E. in Computer Science and Engineering at Sipna COET, Amravati affiliated to Sant Gadge Baba Amravati University, Amravati, Maharashtra, India.

Prof. V. K. Shandilya working as a associate professor in department of Computer Science and Engineering, Sipna COET, Amravati. She completed her M.E[CSE] in 2006 from P.R.M.I.T \& R Badnera, Maharashtra, India and she is having 19 years of working experience. 